\title{
ㄷำ1
}

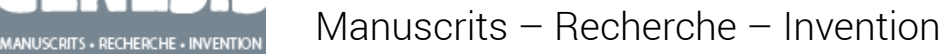

$37 \mid 2013$

Verbal - Non verbal

\section{Des zigzags dans les Pensées de Pascal}

\section{Bruno Clément}

\section{OpenEdition}

\section{Journals}

Édition électronique

URL : http://journals.openedition.org/genesis/1224

DOI : 10.4000/genesis. 1224

ISSN : 2268-1590

\section{Éditeur :}

Presses universitaires de Paris Sorbonne (PUPS), Société internationale de génétique artistique littéraire et scientifique (SIGALES)

\section{Édition imprimée}

Date de publication : 15 décembre 2013

Pagination : 85-90

ISBN : 9782840509196

ISSN : 1167-5101

\section{Référence électronique}

Bruno Clément, «Des zigzags dans les Pensées de Pascal », Genesis [En ligne], 37 | 2013, mis en ligne le 18 mars 2016, consulté le 21 janvier 2021. URL : http://journals.openedition.org/genesis/1224 DOI : https://doi.org/10.4000/genesis.1224 


\section{Des zigzags dans les Pensées de Pascal}

Bruno Clément

pour François Noudelmann

$\mathrm{D}$ ans le tiers inférieur du folio 251 du Recueil original (Copie C1) des Pensées de Pascal ${ }^{1}$, on trouve ces quatre «fragments », le plus souvent publiés ensemble, à la suite l'un de l'autre et dans cet ordre, dans les différentes éditions.

À la transcription de ces quatre " fragments 2 » que proposent D. Descotes et G. Proust dans leur édition électronique des Pensées de $\mathrm{Pascal}^{3}$, je préfère, pour des raisons qui apparaîtront plus bas, la transcription diplomatique que voici :

\section{L'éloquence continue ennuye.}

Les princes et roys jouent quelquefois. Ils ne sont pas toujours sur leurs trônes, Ils s'y ennuieraient. La grandeur a besoin d'être quittée pour être sentie, la continuité dégoûte en tout. Le froid est agréable pour se chauffer.

La nature agit par progrès. elle Itus et reditus, elle $\bar{S}$

passe Et revient puis va plus loin, puis deux fois moins puis plus que jamais etc. zLe flux de la mer se fait ainsi le soleil semble marcher ainsi

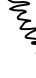

Vous avez mauvaise grâce : excusez-moi s'il vous playe ; sans cette excuse je n'eusse pas aperçu qu'il y eût d'injure révérence parler, il n’y a rien pire de mauvais que leur excuse.

Décidons de numéroter 1, 2, 3 et 4 ces fragments dans l'ordre qu'en propose le Recueil original, et posons que deux choses seulement sont susceptibles de leur conférer une unité (je confesse d'emblée que ma petite note postule l'existence d'une telle cohérence) : soit le thème que transcrivent sans doute à leur façon les deux zigzags, qui se trouvent donc l'un et l'autre dans le troisième fragment (le premier à la fin de sa première ligne, le second constituant comme sa conclusion); soit un autre thème, à identifier, que le zigzag en question (et les quatre fragments qui constituent ce petit ensemble) ne ferait que figurer.

Ces deux graffitis, donc, plus précisément ces deux vue signifier la même chose, le second ne faisant vraisemblablement que donner au premier, énoncé sous une forme générale, une sorte d'universalité méthodique. L'aller-retour qu'il mime est posé non seulement comme un principe naturel, mais comme un principe de «progrès ».

Le zigzag joue ici exactement le rôle que pourrait jouer dans un contexte comparable un schéma physique ou mathématique : après trois ou quatre exemples semblant relever d'un principe unique, dont les effets sont observés dans quelques pratiques et comportements humains (l'éloquence, le sérieux, la grandeur, la chaleur), il intervient pour donner à ces remarques éparses la cohérence d'une loi quasi scientifique. Malgré la diversité

1. On trouvera sur le site <www.penseesdepascal.fr/General/Copies.php > un lien permettant d'accéder à la copie du Recueil original au format PDF basse résolution qu'en propose Gallica : <http://gallica.bnf.fr/ark:/12148/ btv1b9060834h/f147.image.r=pensees+pascal+manuscrit+1601-1700. langFR > . Le folio 251 du Recueil original correspond à la page 147 de ce document. L'ensemble que je m'apprête à considérer constitue dans le classement de Descotes et Proust, le Fragment n 2/7 des Pensées diverses $V$.

2. En vérité, les guillemets conviendraient également au chiffre « quatre », tant il est vrai que l'unanimité fait défaut sur ce point parmi les éditeurs. La transcription de Descotes et Proust signale d'ailleurs que tous les éditeurs (à l'exception de Le Guern et Sellier) dissocient ces fragments présentés par eux comme une unité - au moins matérielle (les chiffres qu'ils donnent entre parenthèses sont ceux de l'édition Lafuma). 3. Voir <www.penseesdepascal.fr/XXVII/XXVII2-savante.php?r1=R\% C3\%A9f\%C3\%A9rence\&r2=\%C3\%A9loquence\%20continue $>$. 


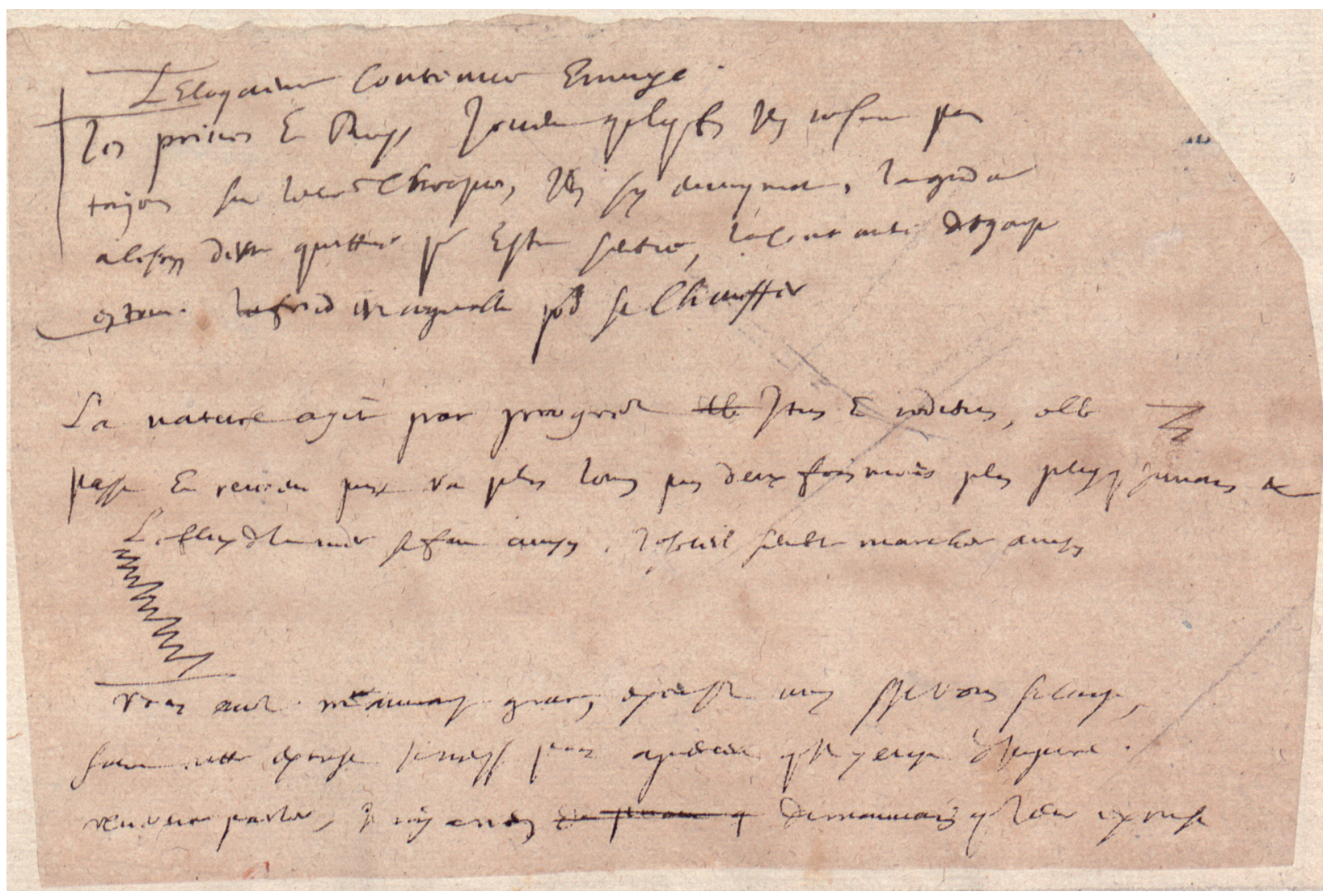

Fig. 1 : Folio 251 du Recueil original des Pensées de Pascal (détail) (BnF, Fr. 9202)

des domaines où elle est observée et en dépit de l'absence de commune mesure apparente entre les éléments évoqués, une unité est postulée, que résume ou que figure le graffiti en zigzag.

On voit mal du coup pourquoi il faudrait à Pascal répéter en fin de fragment le geste qu'il a déjà opéré - et le répéter d'une façon malgré tout légèrement différente.

Mais opéré quand, au fait ? Observons le fac-similé du Recueil original. La transcription diplomatique montre bien que la première version du graffiti n'intervient pas à la fin de la première phrase du fragment, comme le laissent penser presque toutes les éditions de ce fragment, mais à la fin de la première ligne manuscrite, dont elle semble le prolongement.
Cette transcription a encore pour vertu de faire apparaître un détail, que je crois nécessaire à la formulation de mon hypothèse. Il consiste en une rature, à première vue insignifiante.

Dans le manuscrit original en effet on peut voir que Pascal avait d'abord écrit un mot (elle) qu'il a presque immédiatement rayé, mais qui reste lisible entre celui (en langue vulgaire) de «progrès » et celui (en langue savante) de itus. Cette rature est dans un rapport d'homologie frappante avec le propos tenu comme en dehors d'elle. La progression non de la phrase mais de l'écriture de Pascal se fait selon le principe naturel énoncé, la rature constituant le retour (ou reditus) nécessaire au progrès escompté. La plume va 
de l'avant, le discours qu'elle transcrit progresse ; la rature interrompt brièvement ce progrès, dont elle est la condition sûre. Écrire, au fond, est ici une activité naturelle, qui suit du moins ce mouvement observable un peu partout dans la nature par qui a de bons yeux : dans l'éloquence, dans l'habitus des grands, dans la fièvre, dans le mouvement des marées, dans la course du soleil, etc. On a presque l'impression que Pascal découvre sa loi (n'est en mesure du moins de la formuler comme il faut) que lorsqu'il se voit en train de raturer. Le premier zigzag dans le fragment 3 , inscrit pour ainsi dire horizontalement, dans le prolongement de la ligne qui s'achève, est comme la figuration de son progrès.

Le second zigzag, plus long, et dont la configuration est globalement verticale, n'indique semble-t-il pas autre chose. En fin de fragment, inscrit à la suite de tous les exemples évoqués et les englobant tous, il a une valeur quasi conclusive. Il fait par ailleurs étrangement transition avec le fragment suivant, puisque son dernier segment, qui mime le mouvement arrière, est lié au trait horizontal 4 qui le suit, et qui indique qu'il en est malgré tout distinct (un trait semblable, d'ailleurs transcrit par Descotes et Proust, sépare l'un de l'autre les quatre fragments considérés).

Cette liaison, peu contestable, nous contraint à poser loyalement la question de la cohérence. Comment le dernier fragment, qui échappe, au moins à première vue, à la question de la discontinuité, est-il lié (s'il l'est) aux trois autres ? Cela ne va pas de soi. Il s'en faut.

J'ai laissé de côté jusqu'ici l'une des deux hypothèses que je formulais au début. La cohérence dont je fais le pari peut tenir, disais-je, soit dans le graffiti mimant le progrès par itus et reditus, un progrès continu/discontinu à l'image de celui des marées ; soit dans un autre thème, à identifier, dont il conviendrait d'établir le lien avec le premier. Or le thème du progrès par avancées et retraits successifs, s'il convient en effet aux trois premiers fragments, ne semble pas concerner le dernier, qui roule plutôt sur l'efficacité de telle manière de dire ou l'opportunité d'attirer l'attention sur tel point d'un dialogue ou d'une dispute.

Si ce quatrième fragment ne peut être subsumé sous le schéma que constitue les deux zigzags du fragment 3 , il peut en revanche être sans trop de mal mis en rapport avec le premier fragment («L'éloquence continue ennuie »), à condition de considérer que son thème principal n'est pas, cette fois, celui de la discontinuité mais de l'éloquence. Les fragments 1 et 4 ont en effet en commun une préoccupation à l'endroit du bien dire - du mieux dire, du dire au mieux.

C'est chez Pascal, on le sait, une préoccupation majeure. L'un des Opuscules porte précisément sur L'art de persuader. Et Gilberte Pascal, dans la Vie de M. Pascal, qu'elle rédige peu après la mort de son frère, le crédite d'avoir ajouté à son «éloquence naturelle » « des règles dont on ne s'était point encore avisé, et dont il se servait si avantageusement qu'il était maître de son style ». En sorte, précise-t-elle, « que non seulement il disait tout ce qu'il voulait, mais il le disait en la manière qu'il voulait, et son discours faisait l'effet qu'il s'était proposé ». Quant à Léon Brunschvicg, il a cru bon de regrouper dans la première Section de son édition des Pensées tous les fragments ayant de près ou de loin rapport avec cette question de l'éloquence, tant elle lui semblait, dans tous les sens du terme, primordiale.

Si je me tourne donc à présent vers ma seconde hypothèse (celle de l'éloquence comme facteur d'unité des quatre fragments) je dois malgré tout revenir aux deux zigzags du fragment 3 et tenter d'en justifier la présence au regard du dernier fragment, qui s'en prend non pas à une éloquence continue mais - je dirais ainsi les choses - à une éloquence dont la discontinuité est fautive. Une phrase étant prononcée ( "Vous avez mauvaise grâce », par exemple), il ne convient pas d'interrompre le cours qu'elle inaugure, ou qu'elle poursuit, en y insérant, artificiellement, une excuse dont l'effet immanquable serait de ruiner la visée argumentative. Autrement dit, ce serait une faute d'introduire, dans une discussion prétendant à la rationalité, un élément d'éloquence réglée (« la vraie éloquence se moque de l'éloquence », dit aussi Pascal). C'est en effet ainsi que Pascal semble interpréter le procédé qui consiste à vouloir atténuer la portée d'une réserve, voire d'une critique, par une incise de tonalité mondaine sinon éthique. Quelle différence entre cette interruption et les allers et retours dont les fragments 2 et 3 donnent des exemples?

Elle tient en un mot : nature.

4. Cette liaison n'est pas visible dans la transcription de Descotes et Proust, ni dans aucune des éditions qui s'essaient à reproduire ces zigzags (en particulier celles de Le Guern et Sellier) 
Le schéma graphique (le zigzag du fragment 3) n'est ni abstrait ni au fond suffisant. Il indique seulement un mouvement observable dans la nature et qui, à ce titre, pourrait servir de modèle à une éloquence dont Pascal cherche toujours le chiffre. Les fragments 1 et 4 indiquent clairement l'éloquence comme la préoccupation première de ce bref ensemble ; les fragments 2 et 3 , qu'ils embrassent, cherchent à énoncer la loi naturelle dont cette éloquence doit relever et dont le zigzag donne le principe.

Un principe qui n'est pas si simple qu'il en a l'air, pourtant.

Le premier zigzag, je l'ai dit, fait coïncider ce mouvement avec celui de l'écriture. Il semble se féliciter de découvrir, au fil de la plume, que le mouvement de l'écriture, celle-là même qui est en cours, n'est pas en soi condamnable s'il est vrai qu'il est susceptible d'épouser un mouvement partout observable dans la nature. Il justifie du coup la pratique d'écriture.

Le second zigzag, qui s'enchaîne au signe de séparation interfragmentaire, a me semble-t-il une autre portée. Il s'agit cette fois non pas de désigner le mouvement qui, à l'intérieur d'une phrase, lie un mot à un autre, voire une proposition à une suivante, mais dans un raisonnement ou une enquête, une idée à une autre. C'est ainsi en effet que le fragment 4 s'enchaîne au fragment 3 . Il constitue bien par rapport à lui un retour, voire un revirement. Il semble quitter - et quitte en effet la discontinuité comme thème ; mais il n'abandonne pas pour autant la discontinuité comme principe de progrès. Il interrompt en effet le cycle qui semblait bien parti pour continuer à donner des exemples de discontinuité naturelle, constituant à lui seul, une solution de continuité. Moment absolument nécessaire à une progression qu'un esprit soucieux à la fois de rigueur et de naturel puisse raisonnablement légitimer.

La pensée, donc, comme l'éloquence vraie, mais comme aussi le mouvement des mers ou l'évolution d'une maladie (la sclérose en plaques, par exemple) est susceptible d'un mouvement naturel : un progrès par avancées (ou poussées) qu'interrompent, avant qu'ils reprennent de plus belle, des répits, des rémissions, des pauses, des rétractations, des reculs, des retraits, des corrections.

La main qui écrit suit horizontalement le cours de la phrase, qui doit elle aussi se conformer au mouvement naturel de l'épanorthose.

L'épanorthose consiste, selon Fontanier (qui l'appelle aussi « rétroaction »), « à revenir sur ce qu'on a dit, ou pour le renforcer, ou pour l'adoucir, ou même pour le rétracter tout à fait, suivant qu'on affecte de le trouver, ou qu'on le trouve en effet trop faible ou trop fort, trop peu sensé, ou trop peu convenable ». Le zigzag du fragment 3 schématise à merveille, on en conviendra, ce procès rhétorique. Il est remarquable que Fontanier range par ailleurs l'épanorthose au rang des « figures de pensée ». Nous y voilà...

L'esprit qui prétend penser et convaincre procède, pour ainsi dire verticalement, à l'enchaînement des idées ; il doit lui aussi être naturel, mais sans contrevenir à ce principe de correction systématique.

C'est ainsi que fonctionne apparemment ce petit bloc de quatre fragments ; ainsi également que plus généralement procède Pascal lorsqu'il travaille à son chantier en forme de zigzag. Les Pensées de Pascal, il ne faut pas se lasser de le répéter, n'ont jamais existé que dans l'esprit des éditeurs. Éditeurs de papiers («Les Pensées sont les papiers d'un mort », dit très justement Le Guern) qui, dans l'état où on les a trouvés, ne constituent exactement rien qu'un chantier, dont l'intention dernière nous reste à jamais inconnue.

L'hypothèse que je défends ici est donc que ces quatre fragments, semblables en cela à bien d'autres du chantier, ont comme objet et comme principe le souci de « la vraie éloquence »; et que le graffiti en forme de zigzag n'indique rien d'autre que cette exigence d'une soumission de la parole et de la pensée au mouvement naturel qu'il mime.

Si cette hypothèse était avérée, ses implications seraient immenses. D'une conséquence dont il serait, me semble-t-il, dommageable de minimiser les enjeux.

Le zigzag en effet est double et il est susceptible à ce titre d'être envisagé sous deux aspects réellement distincts. Premier aspect, sur lequel je ne reviens pas : l'écriture comme la pensée doivent épouser le mouvement naturel du progrès par allers et retours. Second aspect : une description du monde et des usages humains (ce qu'on a coutume d'appeler l'anthropologie pascalienne) doit elle aussi se conformer à ce mouvement. D'où la question, que je pose brutalement : quel est le statut réel 
de ces notes, thèses et hypothèses anthropologiques qui sont légion, dans les papiers qu'a laissés Pascal ?

On connaît la réponse traditionnelle : l'anthropologie est le pas qu'il convient d'assurer avant de se risquer à la proposition plus proprement religieuse ; il s'agirait de décrire la condition humaine en des termes qui rendent vraisemblable voire incontournable le saut dans la foi. L'anthropologie, en somme, comme tremplin de la théologie.

Notre graffiti bouleverse quelque peu ce schéma reçu. L'anthropologie ne saurait, à s'en tenir du moins à ce bref ensemble de quatre fragments où son rôle est crucial, être tenue pour le préalable à une étape théologique, ou philosophique, encore moins métaphysique. Elle est j'allais dire, elle est seulement - le préalable nécessaire à une démarche éloquente efficace. Connaître l'homme, son esprit, ses arcanes, leur fonctionnement afin de trouver plus efficacement le moyen de les convaincre. L'anthropologie, en somme, au service de la communication. On dira que j'exagère. Et qu'une démarche d'un tel cynisme (on penserait cela, peut-être), ne saurait être imputée à l'auteur des Pensées - ce que Pascal n'a jamais été.

Et pourtant... Et pourtant... C'est Gilberte qui avant moi reconstitue cette logique, sans soupçonner apparemment qu'elle pourrait être imputée à charge au frère tant admiré :

Il concevait l'éloquence comme un moyen de dire les choses d'une manière que tous ceux à qui l'on parle les puissent entendre sans peine et avec plaisir ; et il concevait que cet art consistait dans de certaines dispositions qui doivent se trouver entre l'esprit et le cœur de ceux à qui l'on parle, et les pensées et les expressions dont on se sert, mais que les proportions ne s'ajustent proprement ensemble que par le tour qu'on y donne. C'est pourquoi il avait fort étudié le cour de l' homme et son esprit; il en savait tous les ressorts parfaitement bien ${ }^{5}$.

À supposer que cette connaissance soit sûre (et l'on voit que pour la sœur de Blaise, elle l'était), ses effets seraient immanquables et quelque peu effrayants (Gilberte les expose nûment, et ne cherche pas à cacher son admiration) : face à un tel discours, il serait « impossible à l'esprit de l'homme de ne s'y pas rendre avec plaisir ». Blaise Pascal, nouveau Gorgias...

L'exemple de cet ensemble de fragments, sans doute, est particulier. Peut-être pourtant aurait-on intérêt à en relire un certain nombre d'autres à la lumière de ce zigzag et à soupçonner plus systématiquement, sous une description manifestement anthropologique, une visée décidément rhétorique. On pourrait par exemple citer le fragment suivant pour en tirer une leçon sur le pragmatisme pascalien (c'est la lecture qui est faite du fragment le plus souvent) :

Lorsqu'on ne sait pas la vérité d'une chose il est bon qu'il y ait une erreur commune qui fixe l'esprit des hommes comme par exemple la lune à qui on attribue le changement des saisons, le progrès des maladies, etc., car la maladie principale de l'homme est la curiosité inquiète des choses qu'il ne peut savoir et il ne lui est pas si mauvais d'être dans l'erreur que dans cette curiosité inutile 6.

La portée de la réflexion serait pourtant sensiblement infléchie si on mettait en relation ce fragment avec celuici, qui est en réalité sa suite (il se trouve au verso du même folio dans le Recueil original) :

La manière d'écrire d'Épictète, de Montaigne et de Salomon de Tultie est la plus d'usage qui s'insinue le mieux, qui demeure plus dans la mémoire et qui se fait le plus citer, parce qu'elle est toute composée de pensées nées sur les entretiens ordinaires de la vie, comme quand on parlera de la commune erreur qui est parmi le monde que la lune est cause de tout, on ne manquera jamais de dire que Salomon de Tultie dit que lorsqu'on ne sait pas la vérité d'une chose il est bon qu'il y ait une erreur commune, etc., qui est la pensée de l'autre côté.

Ici aussi, donc, l'anthropologique dissimule un souci rhétorique qui, si l'on se donne la peine de tourner seulement le folio, fait un peu plus qu'affleurer. Telle serait la leçon de notre petit graffiti : soupçonner non pas la portée ou la pertinence du discours anthropologique pascalien, mais sa visée.

\footnotetext{
5. «La Vie de M. Pascal par Mme Périer », dans Blaise Pascal, Euvres complètes, I, Paris, Gallimard, coll. « Bibliothèque de la Pléiade », p. 74. C'est moi qui souligne.

6. Fragment 628 des Pensées (classement de Michel Le Guern), dans Blaise Pascal, Euvres complètes, II, op. cit., p. 798.
} 
Bruno Clément est Professeur à l'université Paris VIII. Il a dirigé le Collège international de philosophie et est actuellement membre de l'Institut universitaire de France. Parmi ses publications : L'Euvre sans qualités. Rhétorique de Samuel Beckett (Seuil, 1994); « De bout en bout », dans Genèses des fins (coll. « Manuscrits modernes », Presses universitaires de Vincennes, 1996) ; L'Invention du commentaire : Augustin, Jacques Derrida (PUF, 2000); Le Récit de la méthode (Seuil, 2005); La Voix verticale (Belin, 2012).

bpe.clement@gmail.com

\section{Des zigzags dans les Pensées de Pascal}

Deux zigzags, presque semblables mais de tailles différentes et disposés en des lieux qui confèrent à chacun une portée spécifique, sont ici mis en relation d'une part avec l'écriture en cours, dont ils semblent mimer le mouvement; et d'autre part avec le progrès de la pensée et de la phrase, supposées suivre un cours plus ou moins naturel. Ainsi, l'anthropologie pascalienne, sujet apparent des fragments considérés, ne serait pas mise au service de l'entreprise théologique ; mais décrite et convoquée pour servir de fondement à une pensée de la « vraie éloquence ».

Two zigzags, almost similar, but of different size and located in places that give each one a specific meaning, are put in perspective here on the one hand with the writing in progress, whose movement they seem to imitate, and on the other with the course of the thought and the sentence, supposed to be a more or less natural movement. Although apparently the topic of these few fragments, the Pascalian anthropology, rather than the first step of the theological enterprise, may be invoked and described as grounds for the thought of "true eloquence".

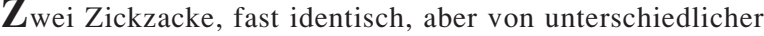
Größe und an Standorten, die jedem von ihnen eine spezifische Bedeutung verleihen, werden hier einerseits mit dem Vorgang des Schreibens, dessen Bewegung sie zu imitieren scheinen, und andererseits mit dem wohl mehr oder weniger natürlich verlaufenden Prozess des Denkens und der Entstehung des Satzes in Verbindung gesetzt. Auf diese Weise stellt sich die als Thema der betrachteten Fragmente erkennbare Pascalsche Anthropologie hier nicht in den Dienst eines theologischen Ansatzes, sondern sie wird beschrieben und als Grundlage für die Idee einer ,,wahren Eloquenz" herangezogen.
Dos zigzags, casi zigzags pero de dimensiones diferentes y dispuestos en lugares que le otorgan a cada uno de ellos un alcance específico, son relacionados, por una parte, con el flujo de la escritura, cuyo movimiento parecen imitar, y por la otra, con el progreso del pensamiento y la frase, que sigue supuestamente un curso más o menos natural. Así, la antropología pascaliana, tema aparente de los fragmentos considerados, no será puesta al servicio de la esfera de lo teológico, sino descrita y convocada para servir de fundamento a un pensamiento de la "elocuencia verdadera".

Dois ziguezagues, muito semelhantes mas de tamanho diferente e situados em lugares que conferem a cada um um alcance específico, são aqui postos em relação, por um lado, com a escrita em curso, a que parecem imitar o movimento, e, por outro lado, com o progresso do pensamento e da frase, que parecem seguir um curso mais ou menos natural. Assim, a antropologia pascaliana, aparente tema dos dois fragmentos, não estaria ao serviço de um empreendimento teológico, mas antes como base para um pensamento de "verdadeira eloquência".

Due zigzag, quasi similari, ma di misura diversa, e collocati in luoghi che danno a ciascuno di essi un rilievo specifico, sono messi in relazione, da un lato, attraverso la scrittura in fieri, il cui movimento sembrano imitare, e, dall'altro, attraverso il procedere del pensiero e della frase, che dovrebbero in principio seguire un andamento più o meno naturale. In tal modo, l'antropologia pascaliana, tema solo apparente di questi frammenti, non sarebbe messa a servizio della teologia, ma esposta in quanto eletta a fondamento del pensiero della "vera eloquenza". 